\title{
Survival with metastases improved by clodronate
}

Long-term follow-up of two British studies has shown that treatment with a first-generation oral bisphosphonate can extend the lives of men with metastatic prostate cancer. By contrast, no benefit was observed among those with nonmetastatic disease. "Our results on overall survival support those obtained for earlier outcome measures in previous studies looking at symptomatic disease progression," notes statistician Matthew Sydes.

During a 12-year period, data were collected from 278 men with metastatic prostate cancer and 471 men with nonmetastatic disease. Participants were randomized to receive sodium clodronate (2,080 mg per day) or placebo, in conjunction with the standard treatment of the time (generally long-term hormone deprivation therapy and/or radiation therapy). All but $7 \%$ of those with metastases died during follow-up; in this group, up to 3 years of clodronate treatment significantly improved overall survival (hazard ratio 0.77). The bisphosphonate had no effect on the survival of men without metastases (hazard ratio $1.12 ; 60 \%$ of participants deceased).

The researchers are now trialing a newer bisphosphonate, zoledronic acid, which is many orders of magnitude more potent than clodronate. "The [STAMPEDE] trial is open in more than 50 centers all across the UK and more than 1,000 men have already been recruited. It will continue to recruit for another 3 years and will also assess the role of chemotherapy (with docetaxel) and a cyclo-oxygenase 2 inhibitor (celecoxib)," reports senior author David Dearnaley.

\section{Kathryn Senior}

Original article Dearnaley, D. et al. Adjuvant therapy with oral sodium clodronate in locally advanced and metastatic prostate cancer: long-term overall survival results from the MRC PR04 and PR05 randomised controlled trials. Lancet Oncol. 10, 872-876 (2009). 